\title{
Brain metastasis in non-small cell lung cancer: we haven't even scratched the surface
}

\author{
Raul Caso, Amber Himmler \\ Department of Surgery, MedStar Georgetown University Hospital, Washington, DC, USA \\ Correspondence to: Raul Caso, MD, MSCI. Department of Surgery, MedStar Georgetown University Hospital, 3800 Reservoir Rd NW, Washington, \\ DC 20007, USA. Email: Raul.Caso@gunet.georgetown.edu.
}

Received: 01 February 2020; Accepted: 15 February 2020; Published: 25 May 2020.

doi: $10.21037 /$ ccts.2020.02.02

View this article at: http://dx.doi.org/10.21037/ccts.2020.02.02

Lung cancer remains the second most common cancer in men and women in the United States with an estimated 229,000 new cases that will be diagnosed in 2020 (1). It is also the leading cause of cancer-related death with an estimated 136,000 lung cancer deaths in 2020 (1). Following resection, 5 -year survival rates for patients with pathological N0 nonsmall cell lung cancer (NSCLC) ranges from $58 \%$ for pT2b to $91 \%$ for pT1a lesions (2). Despite complete tumor resection for pT1-3N0 NSCLC, there remains a relatively high recurrence rate of $13 \%$ (local and distant) over a median follow-up period of 35 months (3). Brandt and colleagues studied 893 NSCLC patients and identified $86 \%$ of the patients that recurred presented with a distant metastasis with $19 \%$ developing brain metastasis at 5 years following complete tumor resection (3). Lung cancer is one of the most common cancers with an accentuated tropism of metastasis to the brain, compared to breast cancer, melanoma, gastrointestinal cancers, and renal cell carcinoma (4). Interestingly, the incidence of brain metastases has risen in the last few decades due, in part, to improved diagnostic modalities and increased patient survival with advanced systemic therapies. Unfortunately, we do not have a clear understanding of what is driving brain metastasis in NSCLC and what the optimal therapeutic approach should be in patients presenting with brain metastases in the era of personalized medicine.

In the last decade there has been an increasing interest in understanding the genetic profile of primary tumors in order to target therapies aimed at individual patients' mutant molecular profiles. DNA sequencing of NSCLC has demonstrated complex genetic heterogeneity, both within primary tumors and between primary and lymph node metastases (5). Unfortunately, because of genetic heterogeneity, targeted therapies aimed at patients' individual tumor genomic profile may not be specific to the genetic profile of metastatic lesions. Recently, Brastianos and colleagues identified genetic heterogeneity between brain metastases and their corresponding primary tumors in a genomic analysis of matched brain metastases, primary tumors, and normal tissue in 86 patients (6). The authors studied 29 lung adenocarcinoma patients. In many cases, potentially actionable clinically relevant alterations were identified in the brain metastases that were not detected in the primary tumor. Overall, in $53 \%$ of cases, the authors identified clinically actionable alterations unique to the brain metastases (6). This finding suggests that genomic heterogeneity between brain metastases and primary tumors may contribute to disparities in intracranial and extracranial disease response to systemic therapies, previously attributed to inadequate blood-brain barrier penetration. Furthermore, the authors studied brain metastases from different intracranial sites in the same patient and identified shared actionable alterations, which suggests homogeneity exists between brain lesions in the same patient (6).

Given these findings, identification of genomic alterations specific to brain metastases and development of targeted therapies against these alterations represents a critical area of research to improve oncologic survival in patients presenting with brain metastases. Unfortunately, in everyday practice, genomic sequencing of brain metastases poses a particular challenge. Most patients presenting with brain metastasis may not be candidates for a resection which limits the amount of tissue available for sequencing. Thus, the extent to which brain metastases from NSCLC share 
the genetic profile of the primary tumor remains unknown, in everyday clinical practice, and a more comprehensive genomic understanding can influence treatment strategies and research directions. Therefore, practical alternative methods of genomic profiling are needed. One such modality is analysis of circulating tumor DNA (ctDNA) in plasma which has shown promise in characterizing tumors and monitoring disease response to therapy (7-9). In one study, mutations unique to the brain metastases were more represented in the cell-free ctDNA from the cerebrospinal fluid (CSF) compared to plasma and the CSF ctDNA was observed to change with therapy (8). CSF ctDNA is a promising modality to identify drug-resistance mechanisms without invasive procedures like a brain biopsy. In a recent study, 341 cancer-associated genes in cell-free DNA isolated from the CSF of 53 patients with brain metastases of solid tumors or primary brain tumors, mutations associated with drug resistance were identified in 4 of 12 patients who progressed in the brain while on therapy (10). These findings suggest CSF ctDNA may facilitate genometargeted treatments in patients with brain metastases and allow treatment surveillance. Further studies are needed evaluating ctDNA extracted from CSF and analyzed using next-generation sequencing techniques to identify all classes of alterations that might be clinically targetable. Ultimately, CSF ctDNA may replace more invasive biopsy procedures in patients with brain metastases.

Immunotherapy has altered the way we treat NSCLC. Significant research has identified the importance of binding of programmed death 1 (PD-1) receptor on activated $\mathrm{T}$ cells by programmed death ligand 1 (PD-L1) on tumor cells leads to T-cell inactivation, resulting in immune tolerance and tumor progression. PD-1 inhibitors, such as nivolumab and pembrolizumab, have been shown to improve survival outcomes in patients with NSCLC $(11,12)$. Unfortunately, the clinical trials for PD-1 inhibitors excluded patients with active brain metastases. Interestingly, a recent study of melanoma brain metastases (MBMs) identified clinically relevant heterogeneity of immune infiltrates in MBMs (13). The authors identified suppression of multiple components of the antitumor immune response in MBMs, such as cytotoxic $\mathrm{CD}^{+} \mathrm{T}$ cells, which are known to correlate positively with responsiveness to $\mathrm{PD}-1$ immunotherapy in melanoma. These findings provide a possible explanation for the relatively low response rates observed with pembrolizumab and nivolumab in melanoma patients with brain metastases $(14,15)$. Interestingly, the authors observed increased immune infiltrates in previously irradiated MBMs.
Similar studies have yet to be performed in brain metastases from NSCLC patients. Future clinical studies perhaps should consider combining radiation and immunotherapy and such studies would evaluate the sequencing and timing of combinatorial approaches to appropriately balance clinical responses and toxicity.

NSCLC patients with brain metastases represent a particularly challenging cohort. There is mounting evidence of the genomic divergence of brain metastases from their corresponding primary tumors which may contribute to the observed disparities in clinical response to various therapeutic regimens. More studies are required, however, to better understand this genomic divergence and identify targetable alterations in primary tumors that may contribute to brain tropism in NSCLC. In addition, disparities in response to immunotherapy may be attributed to significant differences in the immune infiltrates of brain metastases compared to the primary tumor. Overall, the data we currently have suggests brain metastases may not respond to therapy in a similar fashion to the primary tumor. In everyday practice, genomic analysis offers the clinician a better understanding of the clinically targetable alterations unique to the brain metastases, however obtaining tissue biopsy is not routinely feasible. Less invasive approaches, such as CSF ctDNA, which offer critical information required for personalized genomic-directed therapy in patients with brain metastases that are not surgical candidates must be explored.

\section{Acknowledgments}

Funding: None.

\section{Footnote}

Provenance and Peer Review: This article was commissioned by the editorial office, Current Challenges in Thoracic Surgery. The article did not undergo external peer review.

Conflicts of Interest: Both authors have completed the ICMJE uniform disclosure form (available at https://ccts. amegroups.com/article/view/10.21037/2020.02.02/coif). The authors have no conflicts of interest to declare.

Ethical Statement: The authors are accountable for all aspects of the work in ensuring that questions related to the accuracy or integrity of any part of the work are appropriately investigated and resolved. 
Open Access Statement: This is an Open Access article distributed in accordance with the Creative Commons Attribution-NonCommercial-NoDerivs 4.0 International License (CC BY-NC-ND 4.0), which permits the noncommercial replication and distribution of the article with the strict proviso that no changes or edits are made and the original work is properly cited (including links to both the formal publication through the relevant DOI and the license). See: https://creativecommons.org/licenses/by-nc-nd/4.0/.

\section{References}

1. Siegel R, Miller KD, Jemal A. Cancer Statistics, 2020. CA Cancer J Clin 2020;70:7-30.

2. Rami-Porta R, Bolejack V, Crowley J, et al. The IASLC Lung Cancer Staging Project: Proposals for the Revisions of the T Descriptors in the Forthcoming Eighth Edition of the TNM Classification for Lung Cancer. J Thorac Oncol 2015;10:990-1003.

3. Brandt WS, Bouabdallah I, Tan KS, et al. Factors associated with distant recurrence following R0 lobectomy for $\mathrm{pN} 0$ lung adenocarcinoma. J Thorac Cardiovasc Surg 2018;155:1212-24.e3.

4. Berghoff AS, Bartsch R, Wohrer A, et al. Predictive molecular markers in metastases to the central nervous system: recent advances and future avenues. Acta Neuropathol 2014;128:879-91.

5. de Bruin EC, McGranahan N, Mitter R, et al. Spatial and temporal diversity in genomic instability processes defines lung cancer evolution. Science 2014;346:251-6.

6. Brastianos PK, Carter SL, Santagata S, et al. Genomic Characterization of Brain Metastases Reveals Branched Evolution and Potential Therapeutic Targets. Cancer Discov 2015;5:1164-77.

7. Dawson SJ, Tsui DW, Murtaza M, et al. Analysis of

doi: $10.21037 /$ ccts.2020.02.02

Cite this article as: Caso R, Himmler A. Brain metastasis in non-small cell lung cancer: we haven't even scratched the surface. Curr Chall Thorac Surg 2020;2:12. circulating tumor DNA to monitor metastatic breast cancer. N Engl J Med 2013;368:1199-209.

8. De Mattos-Arruda L, Mayor R, Ng CKY, et al. Cerebrospinal fluid-derived circulating tumour DNA better represents the genomic alterations of brain tumours than plasma. Nat Commun 2015;6:8839.

9. Murtaza M, Dawson SJ, Tsui DW, et al. Non-invasive analysis of acquired resistance to cancer therapy by sequencing of plasma DNA. Nature 2013;497:108-12.

10. Pentsova EI, Shah RH, Tang J, et al. Evaluating Cancer of the Central Nervous System Through Next-Generation Sequencing of Cerebrospinal Fluid. J Clin Oncol 2016;34:2404-15.

11. Borghaei H, Paz-Ares L, Horn L, et al. Nivolumab versus Docetaxel in Advanced Nonsquamous Non-Small-Cell Lung Cancer. N Engl J Med 2015;373:1627-39.

12. Herbst RS, Baas P, Kim DW, et al. Pembrolizumab versus docetaxel for previously treated, PD-L1-positive, advanced non-small-cell lung cancer (KEYNOTE-010): a randomised controlled trial. Lancet 2016;387:1540-50.

13. Fischer GM, Jalali A, Kircher DA, et al. Molecular Profiling Reveals Unique Immune and Metabolic Features of Melanoma Brain Metastases. Cancer Discov 2019;9:628-45.

14. Goldberg SB, Gettinger SN, Mahajan A, et al. Pembrolizumab for patients with melanoma or non-smallcell lung cancer and untreated brain metastases: early analysis of a non-randomised, open-label, phase 2 trial. Lancet Oncol 2016;17:976-83.

15. Long GV, Atkinson V, Menzies AM, et al. A randomized phase II study of nivolumab or nivolumab combined with ipilimumab in patients (pts) with melanoma brain metastases (mets): The Anti-PD1 Brain Collaboration (ABC). J Clin Oncol 2017. doi: 10.1200/JCO.2017.35.15_ suppl.9508. 